\title{
EDITORIAL
}

\section{Beware the tyranny, resulted by impact factor mania among the Scientists: a critical review.}

\author{
Dr. C.C. Jayasundara \\ The Librarian, University of Kelaniya, Sri Lanka
}

\begin{abstract}
Publishing articles in high-ranked journals brings a higher payoff that designates a greater likelihood of scholastic success of academics and researchers. In the present climate, it provides many opportunities for researchers to be in a "Golden Club" and catch a great deal of offers from Head-hunters, particularly for jobs, research grants, and consultancies, as many people in the research/academic industry still rely on the fact that greater impact is widely apparent in high ranked journals and the journals with high Impact Factor (IF). In universities, many researchers in academic administration positions, who have already progressed with high visibility by publishing research in journals with high IF, always attempt to put into action the same level of requirements and stipulations in publications for tenure decisions of academic and research staff together with rewarding and endowing perks including financial incentives. Assessing junior colleagues purely by exercising the IF score or journal ranks in senior researchers' mind-set has been widely prevalent in academia in the global context. Enforcing rules and regulations for promotion, recruitment, and financial incentives for tenure decisions only for the publications in high profiled journals with top IF is a kind of mania. It, in turn, creates a dismantle discrimination and precede for academic bullying and professional prejudice in universities. Thus, this paper discusses some of the problems with IF and journal ranking mechanisms, which consist of prevailing distortions in the scientific industry by disclosing the failures to predict the definite impact and creation of perverse incentives. It further proposes some points to be taken for reforming/devising an appropriate mechanism targeting the definite impact of essential scientific values of research and researchers that can be readily used for measuring the impact.
\end{abstract}

Keywords: Predatory publications, IF mania, Publication quality, research manipulation

\section{Introduction}

Western democracy assured a powerful status quo that makes science accessible to the majority of citizens. Scientific communication in this context varies quite widely, from the promotion of science and technology and marketing activities to scientific and technological education and even programmes that help researchers communicate findings in their own and other languages. Also, it was included in this cluster of activities aiming at integrating science into the policymaking processes of modern democracies, which is better known as 'science in society'(Leach, 2015). In the human history, researchers were always more impressed with the number of research-based publications that they could read and use for furthering their investigations to enrich the branches of the universal body of knowledge or informed policy decisions. Many scholarly publications have emerged over the last three decades. The evolution of the review of scientific publications has taken place very influential on the advent of the Internet and the facilitation of the creation of new scholarly journals online. The first journals that appeared in the year 1660, named Philosophical Transactions, Le journal des 
Savants and Miscellanea Curiosa Medico-Physica Academiae Naturae Curiosorum, more than 50 million articles were published, and thousands of scholarly journals have been produced in the scholarly industry.

Taking into consideration the Sri Lankan scholarly industry, the structure of science in Sri Lanka is a joint result of the British colonial regime and subsequent attempts to create independent scientific abilities. Paradoxically, the initial period after independence was characterised by a continuous attempt to eliminate modest neo-colonial influence and set up a local structure to solidly exploit local competencies. Inopportunely, fewer funds, less qualified and competent researchers, and power of destructive political agendas had largely contributed to hampering the attempts to revive scientific capabilities in the country. In such an environment, periodicals in Sri Lanka started in the early 19th century by British scholars in the colonial era. (Yapa, 2011). The Ceylon Government Gazette, published in 1802, was the first periodical published in Sri Lanka. Scientific papers presented at the Ceylon Literary Society were selectively published in the Gazette. The Colombo Journal, the first newspaper published in Sri Lanka in 1832, also contained articles on literary and scientific topics (Senadhira, 1975). The first scientific journal published in Sri Lanka was Medical Miscellany, which was a part of the Journal Ceylon Miscellany, founded in 1853 (Senadhira, 1975). Scholarly publications during the post-independence period gradually moved towards many academic disciplines during the 1980s and 1990s (Yapa, 2011).

When evolving the scholarly publications gradually in the scientific industry, it was needed to measure the impact of research, researchers and prestige of journals, which was termed elite journals in the history. Journal ranking mechanisms are valuable instruments for researchers, policy-makers, and particularly academic administrators to gauge the quality and impact of research and researchers in their organisations. Scientists disseminating research findings in high-ranked journals get higher salaries (Gomez-Mejia \& Balkin. 1992), promotions to higher positions like Professors, Associate Professors, Chief Scientist, etc. There is no exception from this process for the universities in Sri Lanka. Different universities and research institutions, including the promotional marking schemes devised by the University Grants Commission (UGC) in Sri Lanka, have put into action various policies/circulars for recruiting, promoting, and rewarding their researchers in considering the publications in high-ranked journals.

On the other hand, publishing articles in prestigious journals is still perceived as one of the aims of the science in the scientific industry. Many universities worldwide use citation analysis and other matrices for judging the quality of research in publications of their researchers. In many governments, academic and research institutions also consider such measurements for allocating funds for research in universities and other higher education institutions (Adam, 2002; Moed, 2008). The primary mechanism they use is citations as an indicator of scientific worth (Groot and Garcia-Valderrama, 2006; Ventura and Mombru, 2006). These are quantitative in nature, and they imply the position or the significance of a researcher, research, or journal. However, evaluating research and publication is essential for researchers, academics, research and academic institutions, and counties. The quality of scientific contributions is primarily persuasive for resolving the country's burning issues and challenges and developing better interventions. Many researchers have proposed distinctive methods to evaluate the scientific quality of journals. The latter is typically inferred from the citations in scholarly publications which a particular contribution receives. Generally, these principles are used to evaluate scholarly publications. Among them, 
scholarly journals take precedence. The mainstay of all these assessment procedures is evaluating the quality of citations received by a journal, and then the scientific quality is estimated by analysing the networks of scholarly articles by exercising a simple mathematical algorithm.

\section{Background of the Problem}

Generally, quality is considered the essence of scientific research (Johnes, 1988). This quality is vital for human beings as long as the findings are applied to resolve issues, problems and enhance the quality of life. Thus, what new insights have been yielded and how precious such insights are one of the most essential disclose to society. It means whether the research is worthwhile, satisfies stated or implied needs, is free of deficiencies, and meets more human requirements (Nightingale \& Scott, 2007). Over the past decade, scientific communication has been attacked by less quality, predatory journals, as well as fake Impact Factors, which has openly smashed up the credibility of many peer-reviewed scientific journals. The Global Impact Index (GIF) is one of the first fake IFs. The founder of Invisible GIF was actively falsifying several platforms such as "Researchgate" to discuss the benefits of false GIF measurement over the other metrics and disseminating many non-existent and false informational seeds against the Journal Citation Report (JCR) of Thomson Reuter's. 'Citefactor' and Universal Impact Factor (UIF) are also some of the examples of fake IF measurements. However, the unfortunate fact is that some universities and research organisations have defrauded these academic criminals and have added fake brand logos and targeted rankings on their journal pages. Under these circumstances, JCR has been given more weightage by genuine researchers in the scholarly world. However, the issue is here that we cannot consider all the articles in a journal are of high quality and have a significant impact on the world. Thus, the IF of a journal has been yet beyond universal acceptance as it is only one quality indicator. Citation impact gauges scientific utility more than scientific quality (Moed, 2005). The selection of references for articles is subjective and mainly depending upon the preferences of authors. It seems that scientific quality evaluation is a much complex process more than what we currently think through.

Another issue is that a particular paper cited by a researcher does not necessarily mean that it has been read by the author (Donovan, 2006) because no author of his own accord will disclose that article has been cited without reading it. MacRoberts and MacRoberts (2010) have empirically proved that some researchers have cited articles without reading the source. They further presented some examples, i.e. once the identical misprints frequently appear in citations, which imply that such authors have not read the text cited but simply copied from somebody's work. Simkin and Roychowdhury (2005) have extended this empirical evidence with certain percentages that are about $70-90 \%$ citations are cited without referencing the texts but rather coping them from other articles' references. Beed and Beed (1996) explain that the citation counts do not indicate the quality of papers, but it is merely independent of the contested knowledge. Generally, the prestige of a journal is based on two criteria, i.e. scholarly quality and importance. For example, citation counts do indicate which Sociology journals are essential to Sociologists. Nevertheless, Sociologists do not state which journals are of high quality and are not without reading their articles correctly.

On the other hand, they determine the quality and importance as they were taught by their teachers or senior researchers or learned by observing journals published by highly reputable organisations. They may have pre-decided which journals are vulnerable and which journals are prestigious. Thus, it indicates that the citation counts do not directly measure the quality of research, and they are merely subjective, 
dependent and considerably an enumerative measure of the informed judgment of the most excellent, most vulnerable, and most dominant.

In contested disciplines, like Social Sciences, differential citation counts indicate which author, article, or journal accepts and supports the dominant theory at full length and which does not (Lee, 2006). Papers that adopt new and unknown knowledge are anticipated to have insignificant. Thus, differentiation in citation rankings repeatedly reveals the idiosyncratic or philosophical rejection of the theory applied instead of the quality of research or the imperative substance of the research to the general body of knowledge. Hence, the researchers or academics in universities or higher education and research institutions are usually penalised when they are being evaluated for promotions, salary increments, and resea9rch funding, especially for tenure decisions because of the citation rankings and contested findings, which are largely disseminated in journals, not as much as prestigious (Bräuninger \& Haucap, 2003; Lee, 2006).

Many high-profiled researchers worldwide use automated scoring systems to find the impact of research and researchers. The value of research is increasingly assessed by the IF of the journal in which it is published, and this, in turn, influences researchers or academics' ability to climbing up the career ladder. Research and researchers are mainly distressed by an epidemic of this IF mania showed by connecting the research value with the journal, where the work is generally published and disseminated rather than the content of the piece of work itself. IF or journal ranking (JR) has been misused ever more by weighting the publications of each of the others as per the IF of the journal. Despite the widespread popularity that the IF is widely misused, the misuse continues and is likely to continue until most high-ranked researchers and academic administrators stop making unflagging support to enforce the importance of IF or JR for assessing the research and their researchers.

\section{Statement of the problem}

In many higher educational institutions, publications need to appear in flourishing highquality journals with high IF or high ranked journals indexed in prestigious indices like $\mathrm{WoS}$, especially for recruitment and promotions. Besides, there is no exception for this requirement among the academia in Sri Lankan universities. Universities and national bodies responsible for research and development in some countries, including Sri Lanka (e.g. University of Kelaniya, National Research Council, etc.), financially reward researchers for publishing their research in journals with high IFs. Even if the financial incentives have not been initiated by other research organisations, all most all such institutions in Sri Lanka pressure their faculty/researchers to publish research in highranked and indexed journals. Circular 721, 723 916, etc., clearly add more points to indexed journals in WoS according to the professorial hiring and recruitment marking scheme issued by the UGC. However, the question that arises here is how these matrices have misled the academic incentives, funding decisions, and tenure judgments in higher educational institutions. These matrices have made a mania among decision-makers in the entities academic ventures are built. This deceptive mechanism of IF and JR may pursue such decisions in a potentially suboptimal manner, but how can it be mitigated within the organisation itself.

\section{Objective}

The objective of this article is to critically analyse the unscrupulous use of IF and JR systems for the assessment of research and researchers as the tyranny of IF distorts the original objective of the evaluation of research impact. Moreover, it intends to discuss incongruous enforcement of IF 
and JR as it is grossly unfair to the faculty those who are being evaluated and ranked in their profession due to "have-nots" are more prevalent in developing economies, including the economy in Sri Lanka.

\section{Discussion}

\section{Impact Factor and SCImago Journal Rank}

Even though the significance of research outputs can assess quantitatively, institutions in the research industry, including universities, still use the average IF of the journals that researchers have published for research funding allocation and their tenure decisions.

Generally, research impact has many aspects.

1. Changes in access to research;

2. Changes in the extent to which research is considered,

3. Referred to, or read;

4. Citation in documents;

5. Changes in knowledge and understanding;

6. Changes in attitudes and beliefs; and

7. Changes in behaviour (Nutley \& Solesbury, 2003).

The London School of Economics (LSE) defines research impact as "an occasion of influence, and hence it is not the same thing as a change in outputs or activities as a result of that influence, still less a change in social outcomes" (LSE Public Policy Group, 2011:21). However, due to a lack of agreement upon the definitions of research impact, many parities have introduced several methods for measuring the impact. For example, using LSE's definition, the internal or academic impact can be measured by citations in other academic work. In contrast, the external or nonacademic impact can be measured by references in the "trade press or government documents or by coverage in the mass media" (LSE Public Policy Group, 2011:5). Also, qualitative measurements in impact are generally based upon the subjectivity of the peer reviewers, who may have different opinions towards the work based on the values and epistemological paradigms which underpin their thoughts (Yates, 2005).

Several countries have implemented and launched Performance-based Research Funding Systems (PRFSs) depending on either a peer review or a metric-based model (Hicks, 2012). While the Research Excellence Framework in England is one example of a peer review model (Wilsdon et al., 2015), metric-based models have been implemented in mainly Nordic countries, Italy, Belgium, Poland, etc. Amongst metric-based models, Bibliometrics is one of the highly used methods, which has been dominated by the Web of Science by Clarivate Analytics. The WoS devised by Eugene Garfield, who is a worldrenowned scientist and a prominent librarian with an incredible biography, was responsible for a wide range of innovative manuals, including Current Contents, the Science Citation Index (SCI), and other citation databases, the Journal Citation Reports, and Index Chemicus. The journal Impact Factor (IF), developed by Garfield at the Institute for Scientific Information (ISI), reflects the average number of times articles from the journal published in the past two years have been cited in the Journal Citation Reports (JCR) (Garfield, 1999). In addition, Scopus and Google Scholar have also introduced different indices to compete with WoS. The journal IF is widely used in the academic industry as an index to measure the quality of scientific products and is merely based upon citation analyses.

The SCImago journal rank (SJR) indicator is a comparatively novel instrument for evaluating scientific journals that challenge the journal IF in ranking scientific journals on free of charge basis. The main differences between the IF and the SJR originate primarily from disparities in the databases used as the sources of citations and from 
the differences in the methodology of estimation. Scopus, which comprises an extensive collection of scientific journals from many countries and published in different languages, is used for SJR. Thus, SJR is an excellent mechanism that may provide an even more inclusive appraisal of the scholarly estimate of scientific journals. However, the WoS is rather underrepresented, which considers citations from a subset of source journals, exclusive of certain journal publications in languages other than English.

\section{Widespread acceptability of IF indices}

In addition to SJR, SCOPUS has introduced the Source Normalised Impact Factor (SNIF) for measuring the credibility of scholarly journals. These are the most accepted two alternative measures for IF introduced by the Institute for Scientific Information (ISI) and is currently maintained by Clarivate Analytics. However, the credibility of some of the widely popular indices is also questionable. Thus, some countries prepared their list of accepted journals for tenure and recruitment decisions. One example is the University Grants Commission of India. UGC in India has advised all universities and higher education colleges to nominate and promote faculty members based on their new list of approved journals which was composed by the UGC-CARE (Consortium for Academic Research and Ethics), in consulting a team of experts and subject matter experts from various academic disciplines, up to 800 certified journals were included. The previous list had more than 5,000 journals, and nearly 88 per cent of them were identified as predatory or low-quality journals, including 327 SCOPUS indexed journals. Thus, automatically journals available in SCImago are also questionable.

On the other hand, the existing defect of "publish or perish" has detrimentally influenced journal publications' quality. Universities are increasingly demanding more publications for promotions, recruitment and particularly for all kinds of tenure decisions. Further, pressure has increased to publish in journals written in English, even for scholars in some non-English environments. Many large educational institutions in developing economies, including Sri Lanka, mainly focus on teaching but not on research and publications. However, at the time of recruitment, promotions, or tenure decisions, their expectations are beyond reality. They expect many research publications from academics but do not consider the exceeded person-hours of teaching workload over the timehonoured work norms because academic administrators of the universities want to improve the university rankings by raising their brains profiles. Thus, today's academics are being forced by these deplorable pressures to generate more and more journal publications to showcase the laurels of their studious research journey in their careers.

Consequently, instead of publishing all their research results in a single article, many researchers spread out multiple articles from one dataset in making salami research just to supplement their research productivity. Due to high demand from scholars, publishers are also forced to face the challenge by creating new journals that often publish those research articles. These market forces are predominantly apparent in the scholarly publishing industry that will significantly impact the quality of journals. These business pressures are reflected in the growing tendency to treat readers buying the products essentially while maximising potential profit margins of the publishers. Due to the commercialisation of publications, the emergence of mushroom journals designed to capture specific market segments is a direct result of these developments of market pressures in the industry.

The majority of scientists' acceptance and belief yet implies that if your scientific article is printed in a "high impact" journal, the value of the article is tremendously outstanding. Thus, academic administrators always employ the IF for 
estimating the quality of researchers and research sections in institutions/organisations by exercising IF as the most useful mechanism to ascertain the quality of scientists and research, contending that higher the best for high impact. Marder et al. (2010) referred to this situation as "the law of unintended consequences" and suggested that "... reliance on the Impact Factor as a part of the evaluation of candidates and programs has caused myriad problems ...". Also, Editors select scientific papers which will have a higher number of citations. Thus, it seems that journal IF rankings are simply inspired by journals with high-quality papers. However, SJR does not bring any drastic alternative to IF as most of the top 100 journals with IF are also listed in the top-ranked journal list in the SJR.

In analysing SJR and IF's methodologies, the most critical variance between the two methodologies remains that the SJR considers the absolute number together with the "quality" of citations received by a journal. However, IF of the journal takes into account the received citations only in a numerical way. Source Normalized Impact per Paper (SNIP) gauges contextual citation impact of a journal, given that the features of its discipline, particularly the frequency at which scientists cite other articles in their references, the speediness of the maturation of the citation impact, and the degree to which the database employed for the evaluation covers the literature of the discipline. SNIP indicator of Scopus attempts to resolve the issue of SJR, which is the citation behaviour varies among different disciplines and, therefore, leads to systematic differences. However, many normalisation decisions are implicated, which creates an unfeasible gauge for significance (Leydesdorff, \& Opthof, 2010).

While IF has allowed increasing the IF of their journals by promoting self-citations and the predilection for review articles, SJR allows scientists to measure the impact of the journal without the impact of self-citations. Regarding the weight of the different types of articles in the process of calculating the two compared indices of scientific journals, no provision has been made for differentiating between the values assigned to original research articles compared to review articles in any of the indices. Although the SJR was introduced as a substitute to the IF of ISI, which is now called Clarivate Analytics, scientists have not widely accepted it. Thus, as a result of unacceptability, Elsevier was compelled to introduce a new journal metric - CiteScore. However, despite the excessive coverage of CiteScore as it is based on Scopus data, the criteria used for choosing journals is flawed, and the categorisation of inappropriate classification of journals in Scopus has misled the CiteScore calculations.

On the other hand, SJR uses weighted citation scores, i.e. citations receiving from high-ranked journals get more scores than the citations coming from less prestigious journals, which have comparatively a minor network of citations. The IF conversely employs absolute counts. Over time, additional indicators of impact and influence have emerged to address the deficiencies associated with the IF. Many of these new indicators have fallen under the author or article level, which, in addition to citing papers, can also measure how often they are published, downloaded, and generated the number of online comments, etc. Some other metrics have also been created as alternatives to IF, such as Eigen factor, Immediacy Index, Cited Half-Life, Article Influence Score and h-index. However, no matrices have been gained the same attention as IF. One of the significant development of both indices is SJR's scientific, technical and medical journal coverage, of which ISI has not covered many journals. ISI often delays the inclusion of journals for several years, and sometimes it does not include some journals of any kind, though they are highly cited, e.g. some BioMed Central journals. Consequently, many BioMed central 
journals listed in the Scopus are included in the SJR but not in the JCR.

\section{Limitations of the existing impact measurements}

When we try to make a deeper analysis of an article/journal's value, impact and popularity of research, many disparities and limitations of the existing matrices can be found. Coryn (2006) stresses that disciplines, geographical areas, and languages have a somewhat reasonable bias towards certain groups. One example of this partiality is the research in development, where researchers in the global South sometimes do not pay attention to publishing their research papers in high-ranked journals as they may influence development outcomes (Tijssen et al., 2006). Thus, people may think that their research does not have good quality. Consequently, this has become more worries for funding agencies, which seek quality from such matrices. The actual problem is then the researchers think that they should conduct research that matters in their context or whether they should conduct research studies to publish in top-ranked journals (Tijssen et al., 2006).

Many researchers widely criticise the shortcomings of IF for its limitations. They include articles written to editors, editorials, short communications, etc., using only a brief analysis period for the calculation of the IF, and citations that are not included in the computational core of the formula are included in the IF calculation. Factors such as the incorporation of self-citations and a lack of quality assessment of the citation source or the deliberate manipulations of citations by other researchers are also considered weaknesses of the IF. The IF of a journal can sometimes be falsely overstated when the journal often contains review articles, editorials, and letters to editors and also non-inclusion of research published in other languages but not in English. On the other hand, some journal publications publish articles on specific disciplines such as zoological or botanical taxonomies are tend to receive lesser IF as these areas are frequently dependent on old references, and references to taxonomic names do not incorporate into the list of references of the article.

Significantly, citation profiles of research journals are frequently skewed with a small number of highly cited papers which builds the average IF of the whole Journal. Cross (2009) expresses an example of a journal where $40 \%$ of papers were not cited within the IF window, and $80 \%$ of papers had less than the average number of citations that indicates explicit inflation of extensively. This unfortunate situation has been more elaborated by Seglen (1997), and he has found that "... the most cited $15 \%$ of the articles account for $50 \%$ of the citations, and the most cited $50 \%$ of the articles account for $90 \%$ of the citations." He wraps up his study by concluding that "even the uncited articles are then given full credit for the impact of a few highly cited articles that predominantly determine the value of the journal impact factor." The thought-provoking question here is whether we judge the researchers who do not have adequate citation but published their articles in a journal with higher IF.

Sometimes, the number of citations can substantially vary from discipline to discipline, the number of authors, etc. Cross (2009) says that when the number of authors in a particular article in some fields is higher, the number of citations is also higher. It means that highly cited articles tend to contain more authors. He further explains that the basic research fields tend to get higher citation levels than related applied fields that result in higher impact factors. Citation data in the JCR originates only from research indexed by Clarivate Analytics. Thus, interestingly, Seglen (1997) explained this further, expressing that "American scientists who seem particularly prone to citing each other, dominate these databases to such an extent (over half of all citations) as to raise both the citation rate and the mean journal impact 
of American science 30\% above the world average, the rest of the world than falling below average."

Furthermore, a number of citations, H-Index or IF, have now turned into being recognised devices of scientists' research assessment process for financial incentives, selecting top 10 researchers, many academic and research awards, etc in Sri Lanka. UGC Sri Lanka has issued a circular $5 / 2018$ to introduce a transparent h-index-based 5 tier researcher classification scheme to motivate researchers to publish research in reputable journals with higher citations. Nevertheless, the problem here is that this h-index is calculated based on google scholar profiles. Google scholar is not an excellent source to determine someone's h-index. It has many disadvantages, such as the inclusion of non-scholarly citations, double counting of citations, less frequent updating, uneven coverage across disciplines, only electronic sources are considered, and less comprehensive coverage of older publications. Harzing \& van der Wal (2008) argue that the problem of non-scholarly citations and double counting is somewhat limited and attenuated by the use of robust citation metrics such as the hindex. Also, due to the ephemeral nature of google links, the h-index of a researcher may change from time to time, but it should not change that much.

Thus, I do not see it as a successful method of measuring the impact of a researcher and show that the h-index in SCOPUS is more intelligent and logical. Also, appointing and promoting professorial position, the marking scheme based on the UGC circular no. 721, 916, 921 etc., promotes high impact factor journals. The publications in Science Citation Indexed Expanded, Social Sciences Citation Index, or Arts and Humanities Citation Index are given additional 2 points marks for each paper with no maximum limit for the category. The National Research Council (NRC), which provides scientific and research assistance to scientists, contributing to the advancement of scientific knowledge, provides funding for multidisciplinary research aimed at addressing nationally important topics in the form of joint ventures between the private and public sectors that research to strengthen the national economy. Also, it gives presidential Awards for scholarly publications of Scientists to promote them in reaching high-quality research. However, all these attempts still rely on the IF mania as in the academic organisations in the occidental culture. No proper mechanism has been formulated to gauge the decisive impact of research and use of the findings for evidence-based policy decisions in the country. Thus, many social sciences, arts, and humanities researchers are mostly deprived by NRC's mechanism, where their contribution is not well-recognised in Sri Lanka. Therefore, it is needed to free our early career researchers and senior researchers from the tyranny of the IF or number of citations. Thus, the academic administrators and senior researchers, who have been given a task to assess the researchers in our universities, need to keep discipline variations, language disparities, visibility of journals and other factors in their mind before gauging the impact. For example, many senior researchers in the humanities and social sciences cannot compete with the researchers in the sciences. Thus, top researcher awards, financial incentives, and other prestigious awards are always given to scientists and researchers in science and technology disciplines. This has been empirically tested by Williams (2011), claiming "the citation rate in medicine is greater than the cited rate in the social sciences by a factor of 8 to 3 and greater than that in law and the humanities by a factor of 8 to 1 . Physical sciences papers in the WoS are also cited twice as often as those from the social sciences, and four times as often as those in law and the humanities". However, researchers in the humanities and social sciences are still in the continued marginalisation compared to the researchers in the natural sciences due to the 
relegation of humanities and social sciences theory and methodology, the lack of funding of research, and the time for researchers due to increased teaching loads and administration. One example is that the National Science Foundation (NSF) of the Country provides research funding for rigorous research proposals, which have significant implications for the policymaking and the advancement of the scientific body of knowledge but wining proposals in humanities and social sciences are erratic and infrequent.

Why do we have more citations in medical and pure and biological sciences than humanities or social sciences? One of the possible reasons is the research culture. Many research outputs are often published in medicine and pure sciences in journals, but humanities and social sciences are in books and monographs. Indeed, the accuracy of bibliometric analysis for research is often valued to a large extent within the data that contains scientific knowledge. One of the critical issues of the WoS and Scopus is that their publications are mainly in journals but less on other means of disseminating scientific knowledge, such as books, conference proceedings, etc. This can be problematic as scientific communications are extensively inclined by the research field of 'epistemic culture' (Knorr-Cetina, 1991). Initially, articles in Natural Sciences, Engineering and Biomedical research have a wide range of journals compared to disciplines in social sciences and humanities as the journals in these two disciplines are mainly published in native languages. Many articles in most humanities journals cite theories and philosophical issues, and paradigms, typically very old sources. Researchers in this field can also be seen, especially looking at the original source and citing it. Therefore, it appears that authors who publish in humanities journals are being maltreated to some extent. Also, citations are less than natural and medical sciences due to access restraints to the accessible body of knowledge. As a result, the design of scientific work and its implications in science, engineering and biomedical fields available in WoS and Scopus may not be appropriate to compare with social sciences and humanities.

A rigorous culture of a systematic review can also be seen in medicine and sciences, which contains a large number of references for conclusions. For example, the Senate Honours and Cash Prizes at the University of Kelaniya vary depending on the IF of the journals that the researchers publish their research outputs. In this case, many academics with medical and science backgrounds could receive senate honours and cash prizes for having their papers published in high-impact journals. However, the availability of journals in humanities is limited, and their Ifs are also not high. Similarly, the dominance of multidisciplinary journals has an impact on some humanities journals, of which a few have witnessed a shrinkage due to lack of strength to withstand the competition in the market. Thus, the opportunities for humanities researchers to publish their research studies are reasonably limited. In that sense, I believe there is some injustice to humanities researchers in considering IFs in determining monetary rewards.

The significant inadequacy of SJR is though the methodology exercised in the calculation of the SJR may be statistically acceptable, another kind of correspondence such as editorials, letters to the editor, commentaries, perspectives, news, interviews, etc., which may otherwise appeal to the readers or researchers, are substantially underrated in the SJR.

\section{Deliberations in different impact assessments in different countries}

Metrics like IF, SJR has gained higher attraction in the scholarly publication evaluation process as they are straightforward, cheaper, less cumbersome and mostly transparent (Wooding \& Grant, 2003). Even though they have these qualities, most scientists, who are supporters of 
the matrices, agree that they are not the best indicators of research excellence (Andras, 2011).

Usually, two major systems are used in Europe to evaluate research. The first one is that the use of the peer-review process by disciplinary panels. For example, the UK's Research Excellence Framework in which a group of researchers evaluates only selected outcomes. The other method is matrices using different bibliometric indicators such as publication-based or citationbased to measure the outputs of the whole academic community. Many counties like Denmark, Finland, Belgium, Czech Republic exercise this method. In Polish science metric system accepts only JCR's IF. The peer-reviewing process was first introduced in Poland in 1991, but implementation was not successful due to ex-post evaluation, which requires trust and confidence in scientific units and the autonomy of research (Jabłecka \& Lepori, 2009). The government then set the main aim for research institutions that had to educate personnel for industry and the army. The decisions as to which fields of sciences should get the funding were made high on the political level, i.e. they did not depend on evaluating the performance.

The complication here is that some scientists believe research impacts as part of research quality (Yule, 2010; Boaz, 2003;) but others think that quality and impact are two distinct components and contribute to the development of research excellence (Grant et al., 2010). Thus, the emerging issue is, what are the Research Evaluation dimensions of excellence? How to evaluate research excellence? What Criteria to be used? What is the impact of research? Which method to be used... is it Peer Review, bibliometric, or a combination of both? Vieira and Gomes (2015) stress that a bibliometric evaluation relates to an expert-based evaluation in three different ways:

(1) Bibliometrics are used for analysing the expert-based evaluation,
(2) The expert-based evaluation uses bibliometrics as an auxiliary tool, and

(3) The expert-based evaluation is a way of correcting the results of the bibliometric assessment. In Norway, citation-based scores are primarily presented to the panel of experts, who have been appointed to assess the impact of research and research products of scientists and then other information related to journals for gauging the definite impact of research. The problem here is that personal bias can lead to the prejudice of reality by increasing the likelihood of it happening. In countries like Sri Lanka, where there are great ties within the social corporation, there is more contact and relationships, especially between people in the same field. These relationships may have a significant impact on objective decisions and evaluations.

Owing to many inadequacies and limitations in employing citation scores as authentic markers of quality of scientific publications, there is an excellent purpose to ponder some alternative procedures. One important thing that we can see in bibliometric matrices is that many authors cite research available in public domains, and many do not have access to expensive e-journal databases. Thus, referring to open source journals and publicly available articles is comparatively high. Also, self-citation is a matter of fact related to quality assessment, mainly when calculating IF. Citations for a research paper of a scholar is merely depended on the context. Citation patterns depend on many exogenous factors such as the discipline, journal, sub-specialisation, country, availability of the journal, financial capabilities, institutions, subscribers, etc. Thus, the people who have IF mania must look at all these issues when assessing the scholars and their scholarly products because this approach remains highly subjective. Henceforth IF mania is caused to move by the absence of these readily available considerations to ascertain the significance of scholarly article publications. 
The importance of research quality assessment by peers is now very much an alternative methodology of many countries significantly depending on the subject matter rather than using one-dimensional quantitative method and prestige of the publication venue. Researchers must have the power to resist any attempt by institutions to use journal IF for making decisions related to promotion, financial incentives, grant distribution, or tenure decisions because an assessment of the performance of researchers should be mainly focused on contributions rather than publication venue. Scholars should try to combat with IF mania of the academic administrators and attempt to educate them with the deficiencies of IF to measure individual achievement of researchers and be informed of the San Francisco Declaration on Research Assessment (DORA) principles.

We should not try to strictly adhere to a single indicator for assessing the quality of scientific work. According to some scientists, there are many indicators, such as H-index and Eigen factor, which are better than IF. Evaluations that use different metrics are likely to provide more insight than a single method of assessment. In social sciences, references to articles are barely cited within humanities and some sub-domains, permitting such research practically uncited. It has not always been deliberated adequately by other scientists in the same and other disciplines, and it should be a matter of considerable concern among researchers and research administers who are uninformed with the kinetics of citations. Particularly progressive and dynamic areas in research studies include Nanotechnology and Molecular biology, where disseminated research outcomes briefly turn out to be obsolete. Therefore, short-term indices receive a considerable percentage of citations to estimate journal IF. One can argue that field correction that means discipline correction can be applied to journal IF. However, it is not an easy task as many research fields are readily dominated by quite a few journals, in which case adjustments cannot correctly be applied to IF, and then it merely generates relatively IF of unit value (Bordons et al., 2002).

We cannot argue with the utmost confidence that a combination of peers and metrics can make a better ranking approach. In its place, we may advocate different aspects of research quality should be considered for assessments, especially in humanities and social sciences. Social scientists should make a lead to propose an acceptable solution with academic administrators in the university by pressing on to stop the mass euphoria of rankings. On the other hand, the only peer-review process is also not practical. Polish scholars introduced a peer-review assessment system, but the system gave many issues as the evaluation itself was not appropriately planned (Seeber, 2020). The main issue was that peer reviewers already knew that the journals were earlier evaluated, and then they were quite inquisitive to know how many points that previous reviewers have assigned for the journal. Peer reviewers who evaluate many journals in a given period may have experienced the halo effect (Seeber, 2020).

The Australian Research Council follows this latter approach combining both metrics and a review process by "experts in each discipline" (Australian Research Council, 2010). However, both metrics and peer-review methods have been extensively criticised by many scientists. They found that peer reviewer's evaluations and later citations were significantly different (Starbuck, 2006). Peer reviewers were driven too hard and had no adequate time to check each article's quality and journal issue, methodology, or data presented within each research tendered (Hamermesh, 2007).

As an alternative to IF, SJR was introduced. Nevertheless, the method exercised to compute the SJR must be necessary validated, and a number of features may require to be re-examined 
prior to making a rigorous decision to ensure whether its applicability is unflawed over the other matrices. It predominantly appears that the selection of an index is a matter of the fact that the primary concern is connected with the consensus of the evaluators whether their predominant concern is either quality or the popularity of the journal concerned. However, SJR indicates the quartile ranking of each subject discipline where Q1 denotes the top 25\% of journals based on the highest SJR score distribution, Q2 a middle-high position (between top 75\% and 50\%), Q3 a middle-low position (from $50 \%-75 \%$ in the subject category) and Q4 bottom position (bottom $25 \%$ of the SJR scores) (García et al., 2012). Quartile rankings are coined for scientific journals in each discipline according to which quartile of the score distribution the journal subsist in that particular discipline category. It may apply mainly to assessing the quality of journal publications. However, the branches of subject disciplines are much limited, and thus it needs to be taken care of appropriately. That is not an easy task, which can be completed in a year or two. However, the quartile system gives researchers in the humanities and social sciences more advantage than IF. This is because quartiles indicate the divisions according to the merits of journals in that particular field, regardless of IF. Therefore, when granting senate awards and cash prizes, giving more value to articles pushed in Q1 and less value to articles published in Q4 can eliminate the disparity between academic disciplines.

In response to this "impact factor mania", the San Francisco Declaration on Research Assessment condemned the use of "journal-based metrics" as an alternative to funding, recruiting, and promoting individuals or projects. It suggests higher education and research institutions Regarding promotion decisions, especially for researchers at the beginning of their careers, that the scientific content of the article is much more important than the publication matrices or the identity of the journal in the one that was published and consider the value of all research outputs including datasets and software in addition to research publications and wide range of impact measures, including qualitative indicators of research impact that includes the impact on policy and practice. It also recommends to editors - to significantly reduce the focus on journal impact factor as a promotional tool (DORA, 2012).

Researchers now have opportunities in investigating the options of posting in more diverse venues, knowing that their work may still reach the same audience or more audience. Finally, academic administrators should know that although there will always be some prestige associated with publishing in "elite" journals, the journals' quality is in constant change so that wider dissemination will have more citations in publications that have a more significant impact substantial. Also, the impact of research and implications are much more important than the prestige of the journal. Researchers can create full-texts in a digital repository in their organisations or shared ResearchGate portals etc. SHERPA RoMEO offers an online tool to find them. Once archived, the articles will be indexed in academic databases such as Google Scholar. Portals like Figshare and Dryad Digital Repository facilitate access to public data and polished publications, which is increasingly encouraged and encouraged and even mandated by some journals and funders. Also, if the research work addressed the problem of development, it is paramount to reach out the findings to policymakers as all inventions must be communicated, and publication in different formats addressing the categories of different audiences is only a means to that end.

The Howard Hughes Medical Institute is now working to break new ground in its research evaluation practices by only looking at a subset of publications selected to carefully review and 
evaluate (Hatch \& Curry, 2020). I think organisations have to define quality this way. At the same time, some publishers are exploring new practices. For example, PLoS One, a journal published by the Public Library of Science, rates articles only for their technical accuracy and not personally for their potential impact on the field. The underpinning reality is that there are no numerical formulas for evaluating research quality. What matters is the quality of work is ultimately judged by the scientists in the same discipline, which also poses some questions of how scientists review each other's research that should be amicably resolved by the organisation or the scientists in the field. Among the universities in Sri Lanka, the University of Kelaniya has a solid commitment to research and highly encourages researchers to publish works in journals with high impact factors. The University Research Council also assists university academic staff in publishing research-based books that are highly relevant to solving the problem of humanities researchers but merely important to researchers in all fields. Another critical issue in universities is that it is doubtful whether the evaluators of professorial applications make an accurate assessment of the impact of the researchers' research articles and the journals they have published and whether those journals are predatory. As a solution to this problem, the University of Ruhuna seeks its library's assistance to determine the credibility of journals published by the candidates applying for professorial positions. This is very important, and the library can provide evaluators with a report on those journals' credibility prior to evaluation. As long as one does not rigorously gauge this credibility, dependence on IF will be required and will play a more significant role.

\section{Conclusion}

Overall, this critical analysis supports the claims that the unscrupulous use of IF and JR systems for assessing research and researchers as the tyranny of IF distorts the original objective of the evaluation of research impact. It also disclosed that incongruous enforcement of IF and JR is openly unfair to the faculty those who are being evaluated and ranked as "have-nots" are commonly prevalent in many developing nations, including Sri Lanka. Journals may change in quality over time or may cease at a certain point. "Relying on statistics is not more accurate when statistics are improperly used. Indeed, statistics can mislead when they are misapplied or misunderstood" (Joint Committee on Quantitative Assessment of Research, 2008). Thus, it is time for scientists in all disciplines to come together and discuss all these endogenous and exogenous factors and propose a solid solution by combining quantitative and qualitative approaches to demand a fairer and more objective methodology for the assessment of research quality. A project similar to the CARE project implemented by the UGC of India can be built in Sri Lanka. The list was compiled by the UGC based on various protocols and is constantly monitored and updated. In the meantime, researchers in higher education institutions should fight with the academic administration to stop IF mania or JR mania from determining their researchers' coarse core value. Regrettably, the IF has been abused as a device for judging research and researchers' credibility due to numerical values have been misinterpreted or applied inaccurately. Also, a set of academics can engage in a systematic manipulation of citations, which is often termed "citation cartels". Each year, WoS overturns titles and indicates "Editorial Expression of Concern" for journal titles to further examine self-citation patterns. Many intellectuals have already recognised the piles of drawbacks with IF; some attempts have been made to find a substitution, one of which includes gauging impacts other than citations. However, no one has yet achieved the domination of the IF, and its stimulus is likely to continue. There are no shortcuts or numerical formulas for evaluating research quality. However, unless publishers, 
scholars, and institutions make serious efforts to change how the impact of each scientist's work is determined, the scientific community will be hopeless rather than surviving with the numerically driven impact/quality measurements. Until developing a commonly agreed notion towards research evaluation in the global arena, it is intended that this work will draw attention to this issue. That increased education and outreach efforts, like DORA and peer evaluation initiatives, will help academic administrators make better decisions regarding the recruitment, promotions and other tenure track decisions.

\section{References}

Adam, D. (2002). The counting-house, Nature, 415: 726-729.

Andras, P. (2011). Research: metrics, quality, and management implications. Research Evaluation, 90-106.

Australian Research Council. (2010). Excellence in research for Australia 2010: National report. Available at from https://apo.org.au/sites/default/files/resourcefiles/2010-12/apo-nid59933.pdf, Accessed on [12/10/2019]

Beed, C., \& Beed, C. (1996). Measuring the quality of academic journals: The case of economics. Journal of Post-Keynesian Economics, 18, 369-396.

Boaz, A. (2003). 'Evidence-based policy and practice', in Bovaird, T. \& Loffler, E. (eds) Public Management and Governance, London: Routledge.

Bordons, M., Fernández, M., \& Gómez, I. (2002). Advantages and limitations in the use of impact factor measures for the assessment of research performance. Scientometrics, 53(2):195206.

Bräuninger, Michael, \&Justus Haucap (2003), Reputation and relevance of economics journals, Kyklos 56: 175-198.
Coryn, C.L.S. (2006). The Use and Abuse of Citations as Indicators of Research Quality. Journal of Multi-Disciplinary Evaluation, 3(4), 115-120.

Cross, J (2009) Impact factors- the basics. In: The e-resources management handbook (ed. Graham, 5.,) 10.1629/9552448-0-3.17.1, Chapter 18, 1-12.

Cupples, Julia (1918). The R0AE and REF: Resources and Critiques, https://ianpace.wordpress.com/2018/04/03/therae-and-ref-resources-andcritiques/\#: :text=The\%20ranking\%20of\%20jun ior $\% 20$ colleagues,collegiality $\% 2 \mathrm{C} \% 20$ prevent $\%$ 20academic $\% 20$ bullying $\% 2 \mathrm{C} \% 20$ [Accessed on $12 / 08 / 2020]$.

Donovan, Stephen. (2006). Research Journals: Toward Uniformity or Retaining Diversity?. Journal of Scholarly Publishing, 37:230-235. 10.1353/SCP.2006.0009.

DORA (2012). Declaration on Research Assessment. Retrieved from www.ascb.org/dora/ [Accessed on 07/11/2020].

García, J. A., Rodriguez-Sánchez, R., FdezValdivia, J., \& Martinez-Baena, J. (2012). On first quartile journals which are not of highest impact. Scientometrics, 90(3):925-943.

Garfield E. (1999). Journal impact factor: a brief review. Can Med Assoc 161(8):979-80.

Gomez-Mejia, L. \& Balkin, D. B. (1992). Determinants of faculty pay: An agency theory perspective. Academy of Management Journal, 35(5):921-955.

Grant J, Brutscher, P.Q.B., Kirk, S., Butler, L. and Wooding, S. (2010). Capturing Research Impacts: A Review of International Practice. Report prepared for the Higher Education Funding Council for England. Cambridge: RAND Europe. Available at: https://www.rand.org/content/dam/rand/pubs/doc umented briefings/2010/RAND DB578.pdf, [Accessed on 23/12/2019]. 
Groot, T., \& Valderrama, T.G. (2006). Research quality and efficiency - An analysis of assessments and management issues in Dutch economics and business research programs. Research Policy, 35:1362-1376.

Hamermesh, D. (2007). Viewpoint: a replication in economics Can. J. Econ., 40 (3):715-733.

Harzing, A. W. K., \& Van der Wal, R. (2008). Google Scholar as a new source for citation analysis. Ethics in science and environmental politics, 8(1), 61-73.

Hatch, A., \& Curry, S. (2020). Research Culture: Changing how we evaluate research is difficult but not impossible. Elife, 9, e58654.

Jabłecka, Julita, \& Benedetto, Lepori. (2009). Between historical heritage and policy learning: The reform of public research funding systems in Poland, 1989-2007. Science and Public Policy 36(9):697-708.

Johnes, G. (1988). Research performance indications in the university sector. Higher Education Quarterly, 42(1): 54-71.

Joint Committee on Quantitative Assessment of Research (JCQAR) (2008) Citation Statistics, A report from the International Mathematical Union (IMU) in cooperation with the International Council of Industrial and Applied Mathematics (ICIAM) and the Institute of Mathematical Statistics (IMS): available at www.mathunion.org/fileadmin/IMU/Report/Citat ionStatistics.pdf [Accessed on 19/07/2020].

Knorr-Cetina, K. D. (1991). Epistemic cultures: Forms of reason in science. History of Political Economy, 23(1), 105-122.

Leach, J. (2015). The Role of Science Communication in International Diplomacy. In Science Diplomacy: New Day or False Dawn? (pp. 155-169).

Lee, Frederic S. (2006), The ranking game, class and scholarship in American mainstream economics, Australasian Journal of Economics Education, 3:1-41.
Leydesdorff, L., \& Opthof, T. (2010). Scopus's source normalised impact per paper (SNIP) versus a journal impact factor based on fractional counting of citations. Journal of the American Society for information science and technology, 61(11): 2365-2369.

LSE Public Policy Group. (2011). Maximising the impacts of your research: A Handbook for Social Scientists. London: LSE Public Policy Group

MacRoberts, M. and MacRoberts, B. (2010), Problems of citation analysis: A study of uncited and seldom-cited influences. J. Am. Soc. Inf. Sci., 61: 1-12

Marder E, Kettenmann H and Grillner S. (2010). Impacting our young. Proc Natl Acad Sci, 107(50):21233.

Moed, H. F. (2005). Citation analysis in research evaluation. Dordrecht, The

Netherlands: Springer.

Nightingale, Paul \& Scott, Alister (2007) Peer review and the relevance gap: ten suggestions for policy-makers. Science and Public Policy, 34(8):543-553.

Nutley, S., Percy-Smith, J., \& Solesbury, W. (2003). Models of research impact: a cross-sector review of literature and practice. London: Learning and Skills Research Centre

Seeber, M. (2020). How do journals of different ranks instruct peer reviewers? Reviewer guidelines in the field of management. Scientometrics, 122(3):387-1405.

Seglen P.O. (1997) Why the impact factor of journals should not be used for evaluating research? British Medical Journal, 314: 497-508.

Senadhira, A. (1975). The development of science journalism in Sri Lanka' in Libraries and People, Colombo Public Library, Colombo, 261270. 
Simkin, M. \&, Roychowdhury, V. (2005). Stochastic modeling of citation slips. Scientometrics, 62:367-384.

Starbuck, W.H. (2006). The Production of Knowledge. Oxford: Oxford University Press.

Tijssen, R.J.W, Mouton, J.M., Van Leeuwen, T \& Boshoff, N. (2006), How relevant are local scholarly journals in global science? A case study of South Africa, Research Evaluation, 15:163174.

Ventura, O. \& Mombr'u, A.W., (2006). Use of bibliometric information to assist research policy making. A comparison of publication and citation profiles of full and associate professors at a school of chemistry in Uruguay.

Scientometrics 69:287-313.

Vieira, E. S., \& Gomes, J. A. N. F. (2015). The bibliometric indicators as predictors of the final decision of the peer review. Research Evaluation, 25(2):170-183.

Williams, Sierra (2011), Academics shouldn't be afraid that their work may not be being cited as much as they would like: citation rates vary widely across disciplines. Available at https://blogs.lse.ac.uk/impactofsocialsciences/20 11/05/31/citation-rate-variation-acrossdisciplines/ [Accessed on 03/09/2020].

Wilsdon, J., Allen, L., Belfiore, E., Campbell, P., Curry, S., Hill, S., Jones, R., Kain, R., Kerridge, S., Thelwall, M., Tinkler, J., Viney, I., Wouters, P., Hill, J., Johnson, B., (2015). The Metric Tide, Report of the independent review committee on the role of 103 metrics in research assessment and research management. Available at https://blogs.lse.ac.uk/impactofsocialsciences/file s/2015/07/2015_metrictide.pdf [Accessed on 07/08/2020].

Yapa, Geetha (2011). The Historical Development of Sri Lankan Scholarly Journal Publishing (1845 - 2010), Sri Lankan Journal of Librarianship and Information Management, 4(3\&4):59-75.
Yates, L. (2005). Is impact a measure of quality? Some Reflections on the Research Quality and Impact Assessment Agendas. European Educational Research Journal, 4 (4):391- 403.

Yule, G. (2010). The Study of Language (4th eds.) Cambridge University Press. 